\title{
Phase Synchronization between Several Interacting Processes from Univariate Data
}

\author{
N. B. Janson, ${ }^{1,2}$ A. G. Balanov, ${ }^{1,2}$ V. S. Anishchenko, ${ }^{2}$ and P. V. E. McClintock ${ }^{1}$ \\ ${ }^{1}$ Department of Physics, Lancaster University, Lancaster, LA1 4YB, United Kingdom \\ ${ }^{2}$ Department of Physics, Saratov State University, Astrahanskaya 83, 410026, Saratov, Russia
}

(Received 12 October 2000)

\begin{abstract}
A novel approach is suggested for detecting the presence or absence of synchronization between two or three interacting processes with different time scales in univariate data. It is based on an angle-of-returntime map. A model is derived to describe analytically the behavior of angles for a periodic oscillator under weak periodic and quasiperiodic forcing. An explicit connection is demonstrated between the return angle and the phase of the external periodic forcing. The technique is tested on simulated nonstationary data and applied to human heart rate variability data.
\end{abstract}

DOI: 10.1103/PhysRevLett.86.1749

PACS numbers: 05.45.Xt, 05.45.Tp, 87.19.Hh

To ascertain whether or not synchronization exists between two processes in interacting or forced systems represents an important problem of long standing. It has been studied extensively for forced or interacting periodic oscillators [1,2], oscillators influenced by noise [3], chaotic $[4,5]$, and stochastic systems [6]. The synchronization of two coupled periodic oscillators was originally taken to mean that their fundamental frequencies are coincident or rationally connected, and that their instantaneous phases are continuously locked. This definition suggests that one might decide whether the processes are synchronized simply by estimating the Fourier spectrum of the signal from one of the coupled systems and comparing its characteristic frequencies. In the presence of noise, however, only effective synchronization can take place [7] for which the instantaneous phases are only locked during finite times and the frequencies are not necessarily rationally connected. Serious problems may also arise due to the nonstationarity of experimental data.

In view of these difficulties, modern techniques for establishing the presence or absence of synchronization are based on the assumption that the behavior of each subsystem can be considered separately and their individual time series can be compared by a variety of techniques (e.g., by computation of the phase differences between them [5]). This approach can work well for forced systems, or for coupled radiotechnical systems [4], or for biological systems in vitro such as neurons [8]. Cases often arise, however, where the system under study cannot readily be split into its component subsystems in order to measure a separate signal from each of them. In living organisms, for example, noninvasively acquired signals will usually result from the interaction of many processes with different time scales [9]. Thus the question as to whether or not synchronization exists may need to be tackled in cases where one has only a one-dimensional signal to analyze from a system of interacting oscillators.

In this Letter we introduce a novel approach that addresses this problem directly and appears to be widely applicable. It is based on the fact that, where $m$ peri- odic processes interact weakly enough within a single selfoscillatory system, an $m$-dimensional torus will exist in its phase space [2]. Figure 1(a) illustrates the case of two coupled oscillators. If the processes are nonsynchronous, the phase trajectory covers the whole torus surface, and its Poincaré map is a closed curve. But if they are synchronous, stable and saddle cycles lie on the torus, which is then called resonant, and the phase trajectory tends to the stable limit cycle. The Poincare map consists of one or several points of a stable cycle, and the same number of points of a saddle cycle lying between them on the closed curve formed by unstable manifolds of the saddle cycle [Fig. 1(b)]. Consider the dynamics of the Poincaré map, placing the origin in the interior of the closed curve. Each discrete time $t_{i}$ of the trajectory's return to the Poincare secant surface can be described by a phase vector at angle $\phi_{i}$ as illustrated. In the synchronous regime there is a discrete number of possible values of $\phi_{i}$, whereas for the asynchronous one $\phi_{i}$ may take any value within $[-\pi ; \pi]$.

According to Takens' theorem [10] and its extension to noise-influenced systems [11], the system's attractor and its Poincaré map can be reconstructed from a onedimensional time series. In [12] it was shown that the Poincaré map can be reconstructed from return times of the system. Maps for the angles,

$$
\phi_{i}=f\left(\phi_{i-1}\right),
$$

have been applied earlier to reveal determinism in experimental data [13].
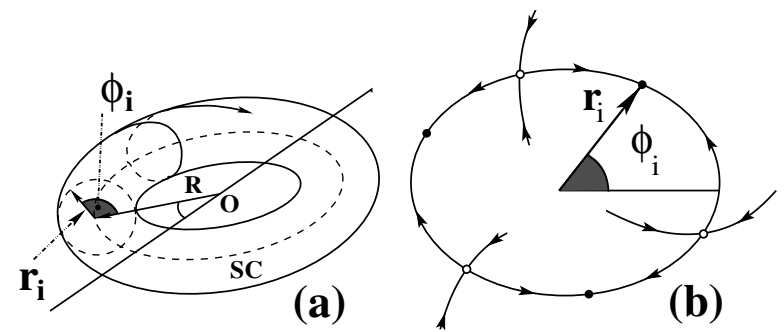

FIG. 1. (a) A two-dimensional torus. (b) Poincaré map for a two-dimensional resonant torus for 1:3 synchronization. 
We now derive the explicit form of the function $f$ in Eq. (1) for the case when the angles $\phi_{i}$ are taken from the return times map of a dynamical system with a twodimensional torus. Consider a quasiharmonic oscillator with eigenfrequency $\omega_{0}$ and amplitude $R$ which is forced harmonically with frequency $\Omega$ and small amplitude $r \ll R$. A two-dimensional torus therefore appears [Fig. 1(a)]. The solution of the resulting nonautonomous equations can then be approximated by a sum of terms describing rotation around some origin $\mathrm{O}$ [Fig. 1(a)] with frequency $\omega$ and amplitude $R$, and around the unstable cycle $S C$ with the external forcing frequency $\Omega$ and amplitude $r$ :

$$
x(t)=R \sin (\omega t)+r \sin (\Omega t) .
$$

The frequency $\omega$ coincides with $\omega_{0}$ in the absence of synchronization; but if the latter exists it is shifted in the direction defined by the forcing. We define the return times as the intervals between successive crossings by $x(t)$ of the threshold $x=0$ in one direction. Although the transcendental equation $x(t)=0$ has no analytic solution, the first term is much larger than the second, so that the times $t_{k}$ of zero crossing by $x(t)$ are close to the times $t_{k}^{*}=\frac{\pi k}{\omega}$ of zero crossing by $R \sin (\omega t)$. We therefore expand (2) as a Taylor series in the vicinity of $t_{k}^{*}$ neglecting terms beyond the linear ones. Taking account of every second zero crossing to register intersections in only one direction, we find the $i$ th moment of crossing,

$$
t_{i} \approx-\frac{r}{R \omega} \sin \frac{2 \pi i \Omega}{\omega}+\frac{2 \pi i}{\omega} .
$$

Denoting the rotation number $\xi=\Omega / \omega$ and setting $\Psi_{i}=$ $\frac{2 \pi i \Omega}{\omega}$, we find the return time

$$
T_{i}=t_{i+1}-t_{i}=2 \frac{r}{R \omega} \cos \left(\Psi_{i}+\pi \xi\right) \sin \pi \xi+\frac{2 \pi}{\omega} .
$$

We place the origin at the central point $\left(\frac{2 \pi}{\omega}, \frac{2 \pi}{\omega}\right)$ of the return times map $\left(T_{i}, T_{i+1}\right)$, introduce the angle $\phi_{i}$ as $\arctan \left[\left(T_{i+1}-\frac{2 \pi}{\omega}\right) /\left(T_{i}-\frac{2 \pi}{\omega}\right)\right]$, and derive an expression for $\tan \phi_{i}$

$$
\tan \phi_{i}=\cos 2 \pi \xi-\tan \left(\Psi_{i}+\pi \xi\right) \sin 2 \pi \xi .
$$

Equation (5) provides an explicit connection between the current angle of the return times map $\phi_{i}$ and $\Psi_{i}$, the latter being the phase of external forcing at the time moment $\frac{2 \pi i}{\omega}$ defining the stroboscopic section of the system. Similarly, we derive an expression for $\cot \phi_{i-1}$ and sum it with Eq. (5) to obtain the following map:

$$
\phi_{i}=\arctan \left(2 \cos 2 \pi \xi-\cot \phi_{i-1}\right) .
$$

Equation (6) shows that, if the forcing amplitude is much smaller than the amplitude of natural oscillations in the system, the map (1) does not depend on amplitudes. The same Eq. (6) can be obtained by consideration of the return times map constructed from the moments when $\dot{x}(t)$ takes zero values, or from the stroboscopic map of the system $\left(x_{i}, x_{i+1}\right)$ where $x_{i}=x\left(\frac{2 \pi i}{\Omega}\right)$. The function $\arctan x$ is defined for $[0 ; \pi]$, although the real angles $\phi_{i}$ lie within $[-\pi ; \pi]$. Thus, we need to extend the map (6) to the interval $[-\pi ; \pi]$ by deducting $\pi$ from $f\left(\phi_{i-1}\right)$ if $\phi_{i-1}$ is less than zero. Further when referring to (6) we will assume the extended map.

Figure 2(a) plots the return function (6) for two values of the rotation number $\xi$ (full curves). The resultant onedimensional map belongs to the class of continuous circle maps [14] of topological degree 1. To reveal how angle $\phi_{i}$ is related to the conventional phase difference $\Phi_{i}$ between the forcing and response, we consider $\Phi_{i}$ at the zero crossing of $x(t)$ [see Eq. (3)]:

$$
\Phi_{i}=-\frac{r}{R}(1-\xi) \sin (2 \pi i \xi)+2 \pi i(1-\xi) .
$$

Calculating rotation numbers $v_{\Phi}, v_{\phi}$ for variables $\Phi_{i}$ and $\phi_{i}$, respectively:

$$
\begin{aligned}
& v_{\Psi}=\frac{1}{2 \pi} \lim _{i \rightarrow \infty} \frac{\Phi_{0}-\Phi_{i}}{i}=(\xi-1) ; \\
& v_{\phi}=\frac{1}{2 \pi} \lim _{i \rightarrow \infty} \frac{\phi_{0}-\phi_{i}}{i}=\xi .
\end{aligned}
$$

Note that $v_{\Phi}$ and $v_{\phi}$ differ by 1 . The points of Fig. 2(a) show the map for angles calculated from numerical data for a Van der Pol system [1] forced by a weak harmonic signal, for two values of the forcing frequency. The simulated data agree well with the theoretical curves.

Next, we consider the general case of a quasiharmonic oscillator forced by $n$ harmonic signals with different frequencies $\Omega_{i}, i=1, \ldots, n$, where the amplitude $A_{i}$ of each of these signals is much smaller than $R$ :

$$
x(t)=R \sin \omega t+\sum_{j=1}^{n} A_{j} \sin \Omega_{j} t, \quad A_{i} \ll R .
$$
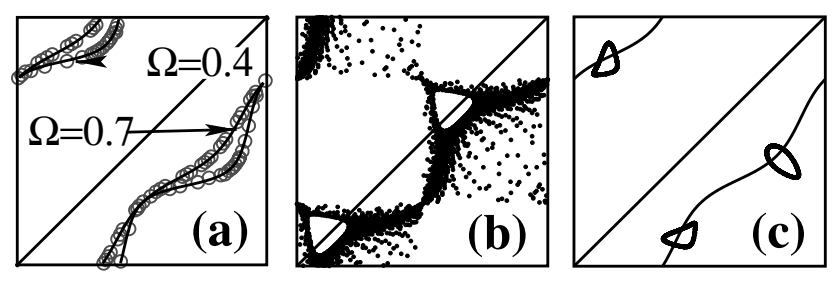

FIG. 2. (a) Return function of the map (6) (curves), compared with the map (1) plotted for numerical data for a weakly forced Van der Pol oscillator (empty circles) with $\omega=1$ and $\Omega=$ $0.4, \Omega=0.7$. The map points fall accurately on the curves. (b) Phase portrait of the map (9) for $\omega=1, \Omega_{1}=0 . \dot{10}, \Omega_{2}=$ 0.010 , i.e., in the absence of synchronization. (c) As in (b), but for $\Omega_{2}=0 . \dot{3}$, showing the effect of $1: 3$ synchronization between $\Omega_{2}$ and $\omega$. The full curve in (c) indicates the return function for (6) with $\omega=1$ and $\Omega=0 . \dot{3}$. 
Denoting $\frac{\Omega_{j}}{\omega}=\xi_{j}$ and $\beta_{j}=\frac{A_{j}}{A_{1}} \frac{\sin \pi \xi_{j}}{\sin \pi \xi_{1}}, \beta_{1}=1$, and proceeding by analogy with the derivation of (6), we obtain a relation connecting two successive angles of the return times map:

$$
\phi_{i}=\arctan \left[2 \cos 2 \pi \xi_{1}-\cot \phi_{i-1}+2 \frac{\sum_{j=2}^{n} \beta_{j} \cos \left(2 \pi i \xi_{j}+\pi \xi_{j}\right)\left(\cos 2 \pi \xi_{j}-\cos 2 \pi \xi_{1}\right)}{\sum_{j=1}^{n} \beta_{j} \cos \left(2 \pi i \xi_{j}+\pi \xi_{j}\right)}\right]
$$

The validity of (9) follows from that of (8). Quasiperiodic motion on an $M$-dimensional torus for $M \geq 3$ is structurally unstable [15], so that after such a torus appears, an arbitrarily small perturbation of the system causes trajectories on its three-dimensional hypersurface to become Lyapunov unstable. Thus, three-frequency quasiperiodic oscillations cannot exist in real systems affected by noise. However, (9) is valid asymptotically as the perturbation tends to zero.

Figures 2(b) and 2(c) illustrate how the map (9) may behave for $n=2$. $R$ is set to 1 . Figure 2(b) shows the phase portrait of the map (9) for the case when no two of the three rhythms are synchronous. In Fig. 2(c) the points belong to the phase portrait of (9) for the 1:3 synchronous regime between the rhythm with frequency $\Omega_{2}$ and the main rhythm, while the full curve shows the return function of (6) for $\omega=1$ and $\Omega=0 . \dot{3}$. Here and in what follows, a dot or dots above one or more digits of a number imply a recurring decimal, i.e., an infinite repetition of the digits under the dots. When all three rhythms are synchronized, the map just consists of a fixed number of points (a trivial case, not illustrated).

Thus, plotting the map (1) for the experimental time series allows one to draw immediate conclusions about the minimum number of rhythms involved, and their synchronization. Although similar conclusions can sometimes be drawn from Poincaré maps, they appear to be less reliable in experimental practice because usually randomness in the instantaneous radii $r_{i}$ [Fig. 1(b)] confuses the picture and prevents one from drawing confident inferences. By comparison, the angular map has a much more pronounced and recognizable structure.

To test the method on a nonstationary process we have simulated the common experimental situation where nonstationarity exhibits itself in a slow random variation of the basic oscillation frequency. We consider the Van der Pol oscillator with the randomly varying parameter $\omega$, under external harmonic forcing:

$$
\begin{gathered}
\dot{x}=y ; \quad \dot{y}=\epsilon\left(1-x^{2}\right) y-\omega^{2} x+C \sin \Omega t ; \\
w=w_{0}+\frac{D_{\eta}}{\tau} \eta(t) ; \quad \dot{\eta}=-\frac{\eta}{\tau}+\mu(t),
\end{gathered}
$$

for $\epsilon=0.1, \omega_{0}=1, C=1 \Omega=0 . \dot{3} . \mu(t)$ is Gaussian white noise $\left(\langle\mu(t)\rangle=0,\left\langle\mu(t)^{2}\right\rangle=1\right)$, and $\eta(t)$ is colored noise of variance $D_{\eta}$ and correlation time $\tau=200$.

First, the presence or absence of synchronization between self-oscillations and forcing was detected by direct computation of the phase difference $\Phi(t)=3 \Phi_{1}(t)-$
$\Phi_{2}(t)$, where $\Phi_{1}(t)$ is the phase of forced oscillations in the system (10), and $\Phi_{2}(t)$ is the phase of the external forcing [5]. In the absence of noise $\left(D_{\eta}=0\right) 1: 3$ phase synchronization occurs, detectable from the plateau around zero on the plot of $\Phi(t)$ over the whole observation time, while the corresponding map (1) consists of just three points (not illustrated here). For noise variance $D_{\eta}=0.1$, nonstationary oscillations take place in the system exhibiting effective 1:3 phase synchronization, detectable from the long plateaus separated by $2 \pi$ phase slips [Fig. 3(a), gray line]. For $D_{\eta}=0.7$, the phase difference shows no plateaus [Fig. 3(a), black line], thus pointing to an absence of synchronization.

Second, efforts were made to analyze the behavior of the system using only univariate data. The power spectra of the synchronized and nonsynchronized regimes [Fig. 3(b)] are very similar (apart from some fluctuational broadening of the peaks), and do not provide a basis for distinguishing between them. However, computation of the map (1) for the two cases provides a much clearer distinction, as shown in Figs. 3(c) and 3(d). Figure 3(c) consists of three isolated segments lying close to a one-dimensional curve (i.e., indicating synchronization) while (d) does not contain isolated segments (i.e., nonsynchronization).

We have tried a preliminary application of the technique to heart rate variability (HRV) data from healthy human subjects, yielding encouraging results. In Figs. 4(a)

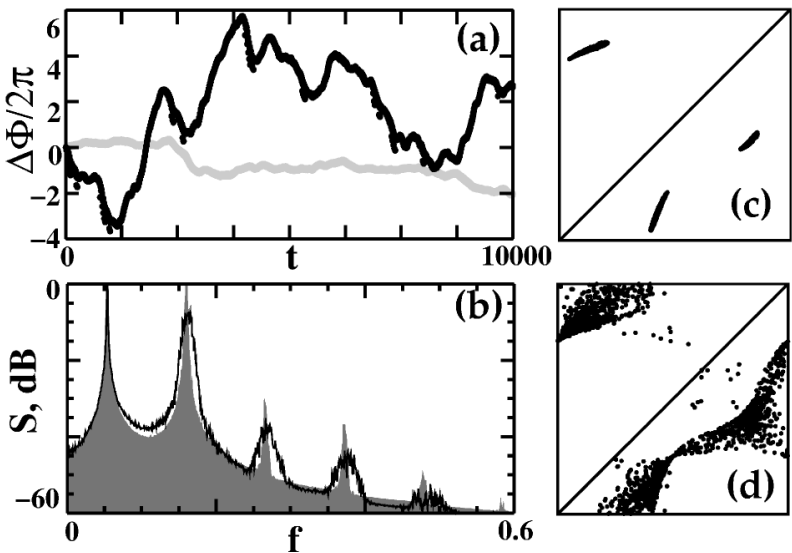

FIG. 3. (a) Phase difference versus time for the forced Van der Pol with slowly randomly varying eigenfrequency (10) for noise variance $D_{\eta}=0.1$ (gray line, showing 2 synchronization plateaus), and for $D_{\eta}=0.7$ (black line, no plateaus). (b) Power spectra of the forced oscillations for $D_{\eta}=$ 0.1 (shaded) and for $D_{\eta}=0.7$ (black line). (c) Angle-of-returntime map for (10) with $D_{\eta}=0.1$ (indicating synchronization). (d) As in (c) but for $D_{\eta}=0.7$ (no synchronization). 

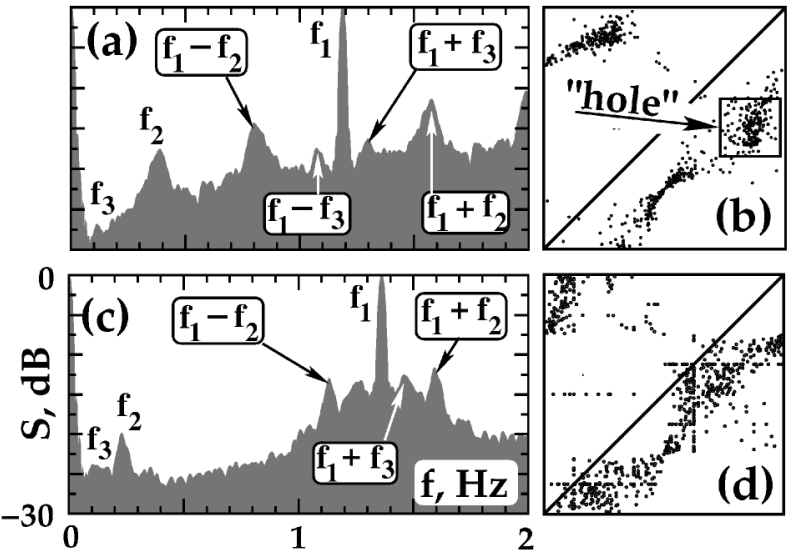

FIG. 4. (a),(c) Power spectra for two sequences of human $R-R$ intervals. Characteristic frequencies and their combinations are indicated. (b),(d) Angle-of-return-time map for these $R-R$ intervals after removing trends. (b) testifies to synchronization between two of the three time scales (see text), while (d) points to the absence of synchronization.

and 4(c) Fourier spectra of the HRV data for the two subjects are given. Three frequencies dominate in the spectra, namely, $f_{1}$ (average heart rate), $f_{2}$ (respiration), and $f_{3}(\sim 0.1 \mathrm{~Hz})$, as well as their combinations. After removing trends in experimental data, we plotted the return times map and the corresponding map (1) [Figs. 4(b) and 4(d)]. These maps, like most others obtained from human HRV data, appear to be in remarkable correspondence with the theoretically predicted dependences [(9); Fig. 2]. Therefore, the first conclusion to be drawn is that the interaction of rhythms within the cardiovascular system of a typical human subject can be considered as weak. Figure 4(b) looks very similar to the phase portrait of map (9) affected by noise for the parameter values as in Fig. 2(c). Three isolated clouds of points testify to the presence of 1:3 phase synchronization between the main heart rhythm and respiration. Actually, each cloud represents a smeared circle (note the "holes" inside the clouds) indicating that the third $(\approx 0.1 \mathrm{~Hz})$ rhythm is present and nonsynchronous with either of the two rhythms mentioned (otherwise instead of circles one would in principle observe smeared points). Figure 4(d) does not imply any synchronization between the rhythms considered.

To summarize, our proposed new approach to the detection of synchronization (or lack of it) between two or more interacting processes is based on an analysis of univariate data only. It has been shown to work well even when the basic frequencies of the interacting processes slowly fluctuate in time due to nonstationarity. Application to human electrocardiograms demonstrated the presence or absence of synchronization between particular oscillatory processes in different subjects.
The work was supported in part by the Royal Society of London, the Engineering and Physical Sciences Research Council (U.K.), and CDRF (REC 006). The authors are grateful to Dr. M. G. Rosenblum and Dr. A. Stefanosvka for helpful discussions.

[1] B. Van der Pol, Radio Rev. 1, 701 (1920).

[2] V.I. Arnold, Geometrical Methods in the Theory of Ordinary Differential Equations (Springer, New York, 1983).

[3] R. L. Stratonovich, Topics in Theory of Random Noise (Gordon and Breach, New York, 1963).

[4] L. M. Pecora and T.L. Carroll, Phys. Rev. Lett. 64, 821 (1990); V. S. Anishchenko, T. E. Vadivasova, D. E. Postnov, and M. A. Safonova, Radiotekh. Elektron. 36, 338 (1991) (in Russian); V.S. Anishchenko, T. E. Vadivasova, D. E. Postnov, and M. A. Safonova, Int. J. Bifurcation Chaos 2, 633 (1992).

[5] M. G. Rosenblum, A. Pikovsky, and J. Kurths, Phys. Rev. Lett. 76, 1804 (1996); A. S. Pikovsky, M. G. Rosenblum, G. V. Osipov, and J. Kurths, Physica (Amsterdam) 104D, 219 (1997).

[6] A. B. Neiman, A. Silchenko, V.S. Anishchenko, and L. Schimansky-Geier, Phys. Rev. E 58, 7118 (1998); A. B. Neiman, Phys. Rev. E 49, 3484 (1994); A. Silchenko, T. Kapitaniak, and V.S. Anishchenko, Phys. Rev. E 59, 1593 (1999); B. Shulgin, A. Neiman, and V.S. Anishchenko, Phys. Rev. Lett. 75, 4157 (1995).

[7] A. N. Malakhov, Fluctuations in Self-Oscillatory Systems (Nauka, Moscow, 1968) (in Russian).

[8] P. Tass, M. G. Rosenblum, J. Weule, J. Kurths, A. Pikovsky, J. Volkmann, A. Schnitzler, and H.-J. Freund, Phys. Rev. Lett. 81, 3291 (1998); A. B. Neiman, X. Pei, D. Russell, W. Wojtenek, L. Wilkens, F. Moss, H. A. Braun, M. T. Huber, and K. Voigt, Phys. Rev. Lett. 82, 660 (1999).

[9] A. Stefanovska and M. Bračič, Control Eng. Pract. 7, 161 (1999).

[10] F. Takens, in Dynamical Systems and Turbulence, Lecture Notes in Mathematics, edited by D. A. Rang and L. S. Young (Springer, Berlin, 1981), Vol. 898, p. 366.

[11] J. Stark, D.S. Broomhead, M.E. Davies, and J. Huke, Nonlinear Anal. Theory Methods Appl. 30, 5303 (1997).

[12] R. Hegger and H. Kantz, Europhys. Lett. 38, 267 (1997); N. B. Janson, A. N. Pavlov, A.B. Neiman, and V.S. Anishchenko, Phys. Rev. E 58, R4 (1998).

[13] K. Suder, F. R. Drepper, M. Schiek, and H.-H. Abel, Model. Phys. 44, H1092 (1998); J. Godelle and C. Letellier, Phys. Rev. E 62, 7973 (2000).

[14] V.I. Arnol'd, Geometrical Methods in the Theory of Ordinary Differential Equations (Springer-Verlag, New York, 1983); J. Guckenheimer and P. Holmes, Nonlinear Oscillations (Springer-Verlag, New York, 1983); L. Glass, Chaos 1, 13 (1991).

[15] S. Newhouse, D. Ruelle, and F. Takens, Commun. Math. Phys. 64, 35 (1979). 